\title{
Динамика поверхностной проводимости в пленках PbSnTe: In с составом вблизи инверсии зон
}

\author{
(C) А.Э. Климов ${ }^{1,2}$, А.Н. Акимов ${ }^{1}$, И.О. Ахундов ${ }^{1}$, В.А. Голяшов ${ }^{1,3}$, Д.В. Горшков ${ }^{1}$, Д.В. Ищенко ${ }^{1}$, \\ Г.Ю. Сидоров ${ }^{1}$, С.П. Супрун ${ }^{1}$, А.С. Тарасов ${ }^{1}$, В.С. Эпов ${ }^{1}$, О.Е. Терещенко ${ }^{1,3}$ \\ ${ }^{1}$ Институт фризики полупроводников им. А.В. Ржанова \\ Сибирского отделения Российской академии наук, \\ 630090 Новосибирск, Россия \\ ${ }^{2}$ Новосибирский государственный технический университет, \\ 630073 Новосибирск, Россия \\ ${ }^{3}$ Новосибирский государственный университет, \\ 630090 Новосибирск, Россия \\ E-mail: klimov@isp.nsc.ru
}

Поступила в Редакцию 24 апреля 2019 г.

В окончательной редакции 29 апреля 2019 г.

Принята к публикации 29 апреля 2019 г.

При гелиевых температурах изучены особенности переходных процессов в эффекте поля в пленках PbSnTe:In с изменением тока до $10^{5}$ раз, качественно соответствующие модели, в которой на поверхности $\mathrm{PbSnTe}:$ In имеется большая концентрация ловушек с различными параметрами. Роль поверхности подтверждается сильным изменением экспериментальных характеристик после химического удаления с поверхности $\mathrm{PbSnTe}:$ In собственных оксидов и ее пассивации слоем $\mathrm{Al}_{2} \mathrm{O}_{3}$.

Ключевые слова: твердый раствор PbSnTe:In, эффект поля, поверхностная проводимость, МДП-структура.

DOI: 10.21883/FTP.2019.09.48125.08

\section{1. Введение}

В твердых растворах $\mathrm{Pb}_{1-x} \mathrm{Sn}_{x}$ Те при $x>0.3-0.35$ происходит инверсия валентной зоны и зоны проводимости с переходом в состояние топологического кристаллического изолятора (ТКИ) [1,2] с появлением поверхностных состояний, имеющих дисперсию в виде конуса Дирака. Особый интерес представляют транспортные свойства ТКИ, обусловленные этими поверхностными дираковскими состояниями. Однако данные по исследованию поверхностного транспорта PbSnTe с помощью эффекта поля (ЭП) практически отсутствуют как для ТКИ-составов, так и для PbSnTe с нормальным энергетическим спектром. Использование ЭП существенно затруднено из-за большой статической диэлектрической проницаемости PbSnTe. Для указанных составов при низких температурах $\varepsilon_{\mathrm{PbSnTe}}>2000$ [3]. По сравнению с другими полупроводниками такая величина $\varepsilon$ ведет как к уменьшению напряженности внешнего электрического поля $E$ на границе с PbSnTe до ста и более раз, так и к соответствующему уменьшению экранирующей $E$ поверхностной плотности носителей заряда $\Delta n_{s}$. Так, например, для $E=1.5 \cdot 10^{6} \mathrm{~B} / \mathrm{cm}$ при $\varepsilon_{\mathrm{PbSnTe}} / \varepsilon_{g}>100$, где $\varepsilon_{g}$ - диэлектрическая проницаемость подзатворного диэлектрика в МДП-структуре, $\Delta n_{s}<8.3 \cdot 10^{8} \mathrm{~cm}^{-2}$. Даже если плотность локализованных поверхностных состояний будет пренебрежимо мала по сравнению с $\Delta n_{s}$, изменение полной проводимости образцов толщиной 1 мкм окажется всего около $1 \%$ при концентрации $n_{0} \approx 10^{15} \mathrm{~cm}^{-3}$. Однако известно, что в силу особенностей фазовых диаграмм, электрической активности и свойств собственных дефектов кристаллической решетки такие значения $n_{0}\left(p_{0}\right)$ практически не реализуемы ни для каких составов $\mathrm{PbSnTe}$.

Ситуация меняется с добавлением индия в $\mathrm{PbSnTe}$ определенного состава. В таком случае при гелиевых температурах может быть реализовано даже изолирующее состояние PbSnTe:In [4]. Однако для ЭП появляется характерная для $\mathrm{PbSnTe}: \mathrm{In}$ проблема высокой концентрации сравнительно „медленных“ ловушек, в том числе вблизи или на поверхности. В принципе снижение влияния таких ловушек на величину ЭП может быть достигнуто как разработкой соответствующей технологии подготовки и пассивации поверхности, так и за счет достаточно высокой скорости изменения $E$, при которой значительная часть ловушек не будет успевать перезаряжаться. Исследование ЭП в PbSnTe:In в таких условиях и являлось целью настоящей работы.

\section{2. Образцы и методика эксперимента}

Исследовались пленки $\mathrm{Pb}_{0.71} \mathrm{Sn}_{0.29} \mathrm{Te}: \mathrm{In}$ толщиной 1.7 мкм, полученные методом молекулярно-лучевой эпитаксии МЛЭ на подложке (111) $\mathrm{BaF}_{2}$. Концентрация электронов составила $n_{0}<10^{13} \mathrm{~cm}^{-3}$ при $T<20 \mathrm{~K}$, максимальная подвижность $\mu \approx 3 \cdot 10^{4} \mathrm{~cm}^{2} \cdot \mathrm{B}^{-1} \cdot \mathrm{c}^{-1}$ при $T \approx 40 \mathrm{~K}$. Схема экспериментальной структуры показана на рис. 1 вместе с принципиальной измерительной схемой. Две контактные области (исток и сток) $n^{+}$-типа проводимости к высокоомной области $i$-типа были сформированы напылением в вакууме слоя 


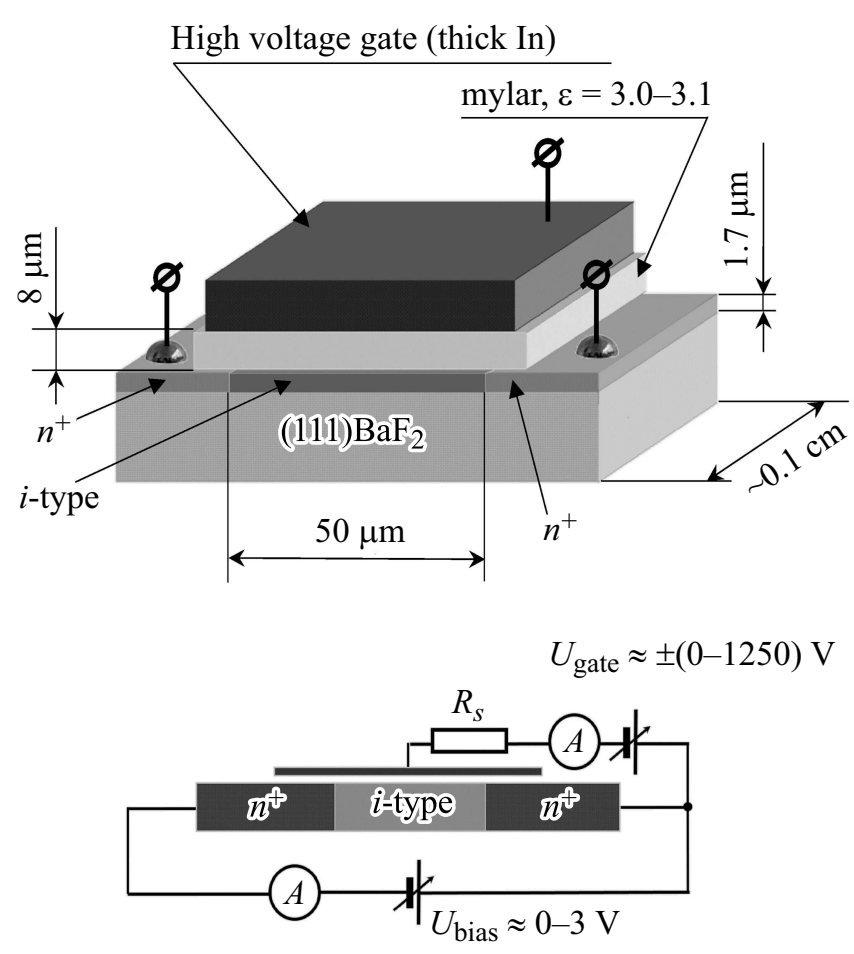

Рис. 1. Схема экспериментальной структуры (вверху) и принципиальная измерительная схема (внизу).

In толщиной $\sim 200$ нм с последующим диффузионным отжигом. Расстояние между контактами составило 50 мкм, протяженность контактов в зазоре $0.1 \mathrm{cм}$. Как и в работе [5], в качестве изолятора в МДП-структуре была использована майларовая пленка толщиной 8 мкм. Это позволило нам измерить затворные характеристики одного и того же образца до и после обработки поверхности PbSnTe:In, находящейся под затворным майларовым диэлектриком, и сравнить их между собой. На расположенный над $i$-областью затвор подавалось напряжение $-1250 \mathrm{~B}<U_{\text {gate }}<+1250 \mathrm{~B}$ (электрическое поле $\left.-1.56 \cdot 10^{6} \mathrm{~B} / \mathrm{cm}<E<+1.56 \cdot 10^{6} \mathrm{~B} / \mathrm{cm}\right)$. На рис. 2 приведены температурные зависимости концентрации и подвижности электронов как в области $i$-типа проводимости, так и в областях $n^{+}$-типа проводимости, рассчитанные из измерений эффекта Холла в магнитном поле $B=0.2$ Тл. Из рисунка видно, что в области $i$-типа проводимости при $T=20 \mathrm{~K}$ концентрация электронов $n_{0}<10^{13} \mathrm{~cm}^{-3}$, а при более низких температурах она становится не измеряемой из-за высокого сопротивления образцов. Высокая подвижность электронов свидетельствует о достаточно хорошем качестве исследованных монокристаллических слоев PbSnTe:In.

Обработка поверхности пленки с целью удаления собственных оксидов проводилась в течение $10 \mathrm{c}$ в растворе $\mathrm{HCl} / \mathrm{CH}_{3} \mathrm{CH}(\mathrm{OH}) \mathrm{CH}_{3}$ (HCl-iPA), представляющем собой изопропиловый спирт, выдержанный в парах $\mathrm{HCl}$ в течение суток [6], с последующей промывкой в чистом $\mathrm{CH}_{3} \mathrm{CH}(\mathrm{OH}) \mathrm{CH}_{3}$. Время между такой обра- боткой поверхности и загрузкой образца в вакуумную камеру для нанесения пассивирующего слоя $\mathrm{Al}_{2} \mathrm{O}_{3}$ толщиной 72 нм методом атомно-слоевого осаждения [7] не превышало 10 мин.

Измерения проводились в металлической камере, экранированной от фонового излучения, расположенной в жидком гелии или в его парах при $T=4.2 \mathrm{~K}$. В качестве источника освещения использовалась миниатюрная вакуумированная безцокольная лампа накаливания [8].

При температуре жидкого гелия $T=4.2 \mathrm{~K}$ были исследованы квазистационарные вольт-амперные характеристики экспериментальной структуры при $U_{\text {gate }}=0$, а также при $U_{\text {gate }}=-1250$ В и $U_{\text {gate }}=+1250$ В. Были исследованы временны́е зависимости тока исток/сток при фиксированных значениях напряжения исток/сток $U_{\text {bias }}$ и различных скоростях изменения как положительного, так и отрицательного $U_{\text {gate }}$. Такие измерения были проведены как без освещения, так и при освещении образца в области фундаментального поглощения. Были исследованы временны́е зависимости тока исток/сток при

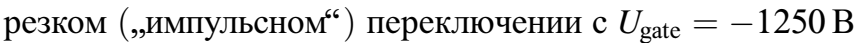
на $U_{\text {gate }}=+1250 \mathrm{~B}$ и обратно, затворные характеристики при различных скоростях изменения $d U_{\text {gate }} / d t$ при фиксированных значениях $U_{\text {bias. }}$ Была исследована трансформация ряда экспериментальных характеристик МДП-структуры при увеличении температуры от $T=4.2 \mathrm{~K}$ до $T=25 \mathrm{~K}$. Далее приводятся некоторые из полученных экспериментальных зависимостей и их обсуждение.

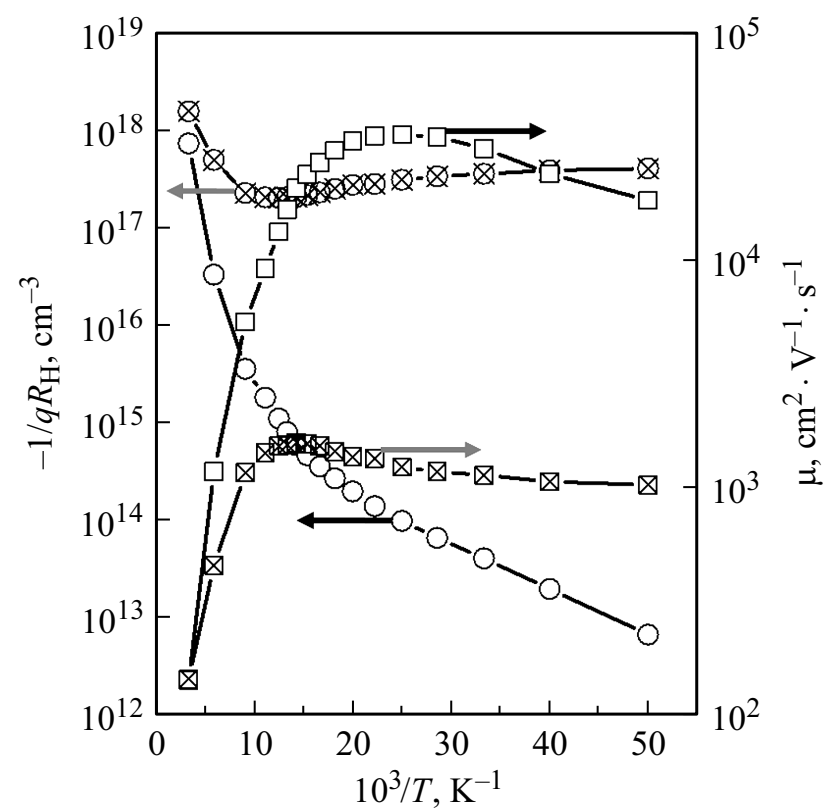

Рис. 2. Температурные зависимости рассчитанных по эффекту Холла концентрации (левая ось ординат) и подвижности (правая ось ординат) электронов в области $i$-типа проводимости (открытые круги и квадраты) и в областях $n^{+}$-типа проводимости (перечеркнутые круги и квадраты). 


\section{3. Результаты и обсуждение}

В области $U_{\text {bias }}=0.02-3.0$ В ток исток/сток менялся в диапазоне $I=10^{-11}-10^{-3}$ А. Зависимости $I=f\left(U_{\text {bias }}\right)$ соответствовали режиму токов, ограниченных пространственным зарядом. На рис. 3 в качестве примера влияния на $I$ скорости изменения $U_{\text {gate }}(t)$ показаны затворные характеристики $I=f\left(U_{\text {gate }}\right)$ для 2 значений $d U_{\text {gate }} / d t$. Помимо сильно отличающейся формы гистерезисных петель, для $d U_{\text {gate }} / d t=25 \mathrm{~B} / \mathrm{c}$ наблюдаются особенности типа "переключений“, начинающиеся (конец стрелок на верхнем рисунке) при $U_{\text {gate }} \approx-500$ В и $U_{\text {gate }} \approx+500 \mathrm{~B}$. Наблюдавшиеся трансформации ЭП с ростом $T$ дают основания предполагать, что при $T=4.2 \mathrm{~K}$ образцы находятся в сегнетоэлектрическом состоянии, которое может быть причиной таких „переключений“.

На рис. 4 показаны зависимости тока $I(t)$ для разных полярностей $U_{\text {gate }}$, но одинаковых временны́х зависимостей $\left|U_{\text {gate }}(t)\right|$ и напряжения на источнике освещения $U_{\text {light }}(t)$. Видно, что зависимости отличаются кардинально. Моменты включения и выключения освещения отражены на верхнем рисунке (правая шкала). Например, помимо радикального отличия $I(t)$ для положительного и отрицательного $U_{\text {gate }}$ до включения освещения (отличие
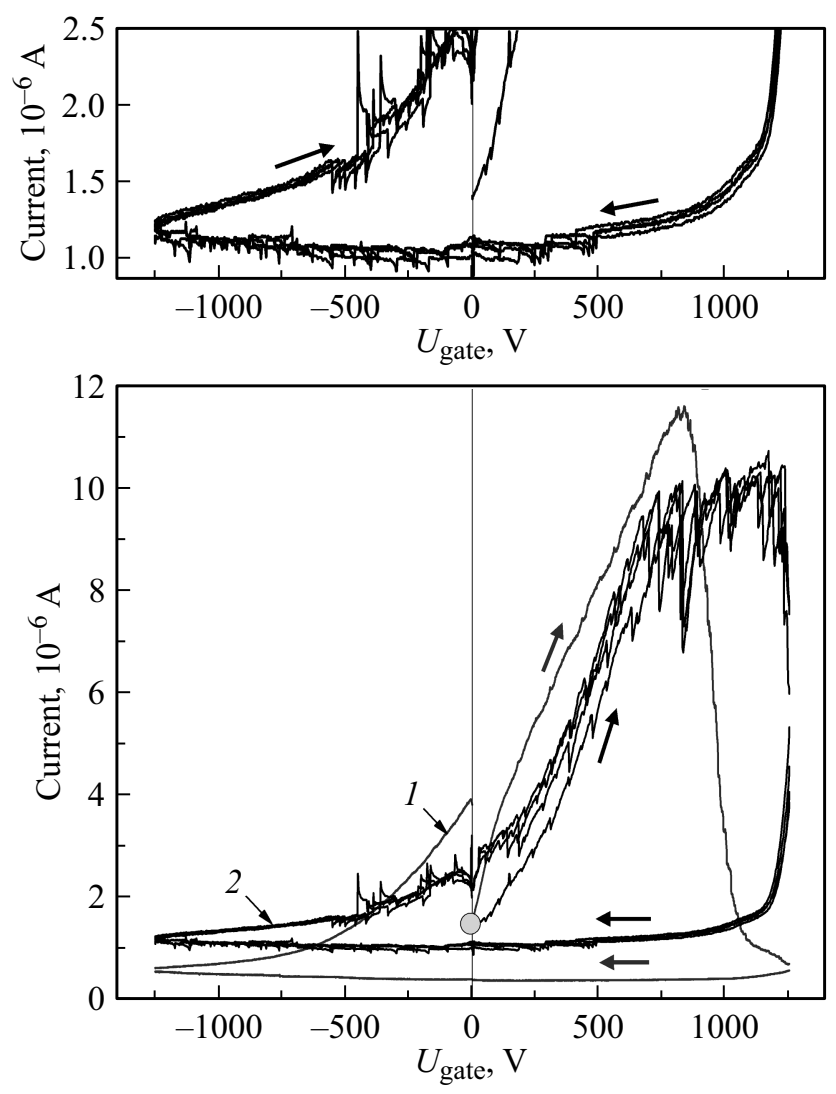

Рис. 3. Внизу - зависимость тока исток/сток от затворного напряжения для $d U_{\text {gate }} / d t, \mathrm{~B} / \mathrm{c}: 1-2.5,2-25$ (четыре „прохода“"). Вверху - та же зависимость для $d U_{\text {gate }} / d t=25 \mathrm{~B} / \mathrm{c}$ в увеличенном масштабе. Направление изменения $U_{\text {gate }}$ показано стрелками. Напряжение исток/сток $U_{\text {bias }}=0.72$ В.
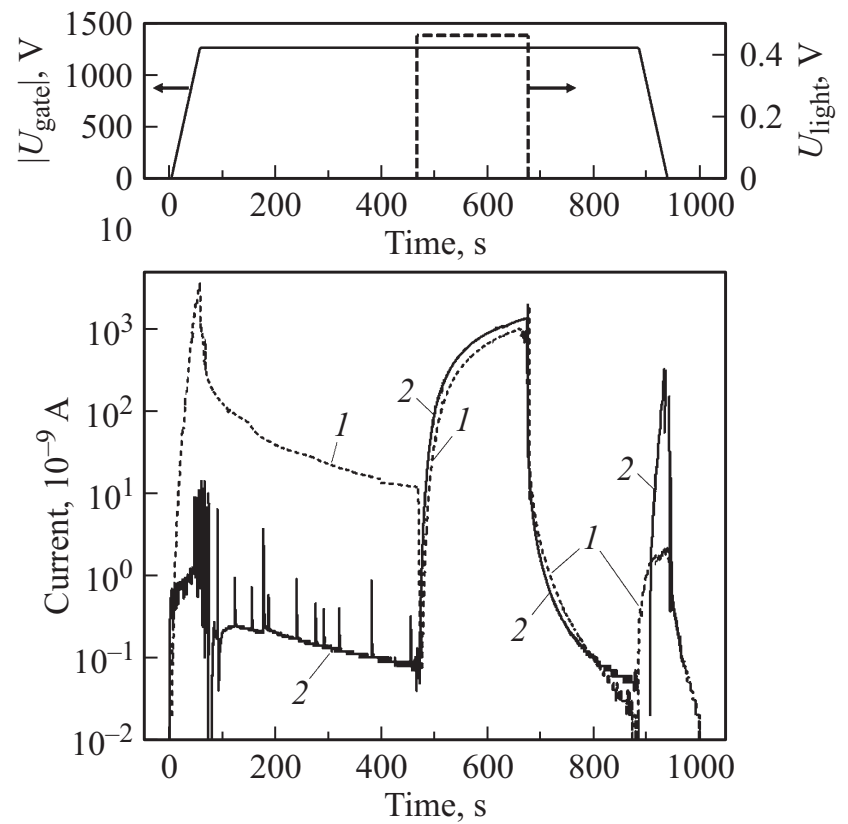

Pис. 4. Зависимость тока исток/сток от времени (внизу) при положительном (1) и отрицательном (2) затворном напряжении $U_{\text {gate }}$ и $U_{\text {bias }}=0.091$ В. Вверху показаны временны́е зависимости абсолютного значения $U_{\text {gate }}$ (левая шкала) и напряжения на источнике освещения образца (правая шкала).

тока до $10^{4}-10^{5}$ раз), в момент „включения“ освещения вблизи $t=470$ с на верхней кривой $\left(U_{\text {gate }}>0\right)$ наблюдается „отрицательная фотопроводимость“, при уменьшении $\left|U_{\text {gate }}\right|$ в области $t>880$ с „всплеск“ тока для $U_{\text {gate }}<0$ примерно в 100 раз больше, чем для $U_{\text {gate }}>0$. На качественном уровне это соответствует модели, в которой внешнее поле $E$ экранируется как подвижными носителями заряда в объеме, так и носителями, локализованными на поверхности PbSnTe:In на ловушках с разными параметрами. Зависящее от условий измерения ЭП заполнение таких ловушек и определяет сложную динамику наблюдаемых эффектов.

На рис. 5 приведены по две затворные характеристики МДП-структуры, измеренные до обработки поверхности в HCl-iPA и после такой обработки с последующим нанесением пассивирующего слоя $\mathrm{Al}_{2} \mathrm{O}_{3}$ толщиной 72 нм. Видно, что изменение состояния поверхности вследствие указанных воздействий количественно и качественно изменило затворные характеристики. Так, вблизи $U_{\text {gate }}=-1250$ В ток уменьшился на $1.5-2$ порядка. При уменьшении затворного напряжения от $U_{\text {gate }} \approx 1000$ В до $U_{\text {gate }}=-1250$ В до обработки ток менялся слабо, в то время как после нее он в этой же области $U_{\text {gate }}$ уменьшается до 10 раз. При этом исчезла серия особенностей, хорошо видных на рис. 3 вверху, но появилась ранее не наблюдавшаяся серия „выбросов“ тока. Заметно изменилась величина гистерезиса в области положительных значений затворного напряжения. Хорошо видное в этой области до обработки большое число 

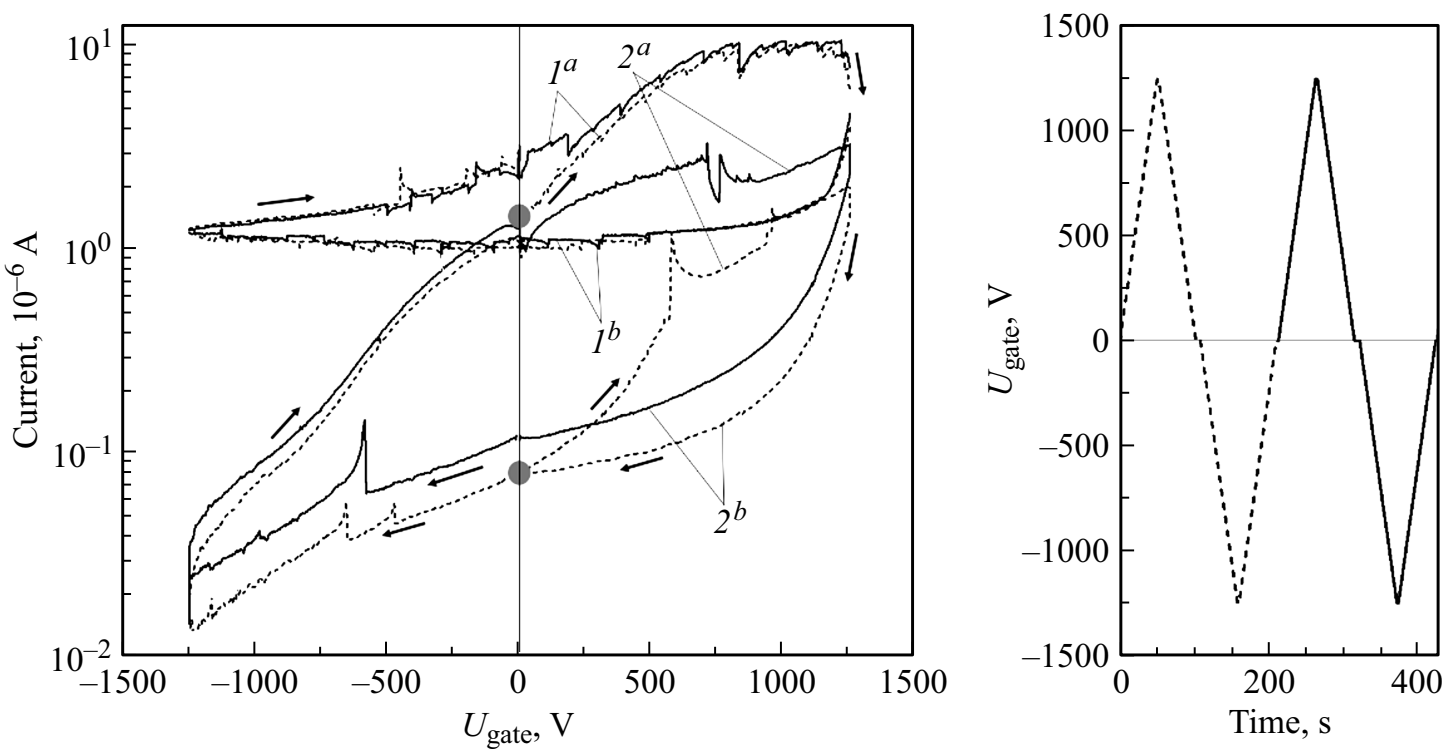

Рис. 5. Слева - зависимости тока исток/сток от затворного напряжения $U_{\text {gate }}$, справа - зависимость $U_{\text {gate }}$ от времени. Напряжение исток/сток $U_{\text {bias }}=0.72 \mathrm{~B}$. Скорость изменения затворного напряжения $\left|d U_{\text {gate }} / d t\right|=25 \mathrm{~B} / \mathrm{c}$. На левом рисунке: $1-$ до обработки поверхности PbSnTe:In, 2 - после удаления окислов и нанесения $\mathrm{Al}_{2} \mathrm{O}_{3}$. Направление изменения $U_{\text {gate }}$ показано стрелками возле кривых и отображено индексами: $a-$ увеличение, $b-$ уменьшение $U_{\text {gate. }}$ Кругами на левом рисунке при $U_{\text {gate }}=0$ показаны начальные точки измерений (на правом рисунке соответствует моменту $t=0$ ). Пунктирные кривые соответствуют первым циклам измерений (на правом рисунке $t=0-210 \mathrm{c})$, сплошные - вторым $(t=210-430 \mathrm{c})$.

особенностей при росте $U_{\text {gate }}$ исчезло после обработки, а вместо него появилось небольшое число характерных „переключений“, не наблюдавшихся до обработки.

Таким образом, видно, что величина и характер изменения тока под действием затворного напряжения сильно зависят от состояния поверхности. При этом сама величина изменения тока исток/сток под действием затворного напряжения достигает нескольких порядков (до $10^{5}$ раз в некоторых режимах). Следовательно, можно считать установленным, что если и не все, то многие специфические релаксационные процессы в исследованной пленке $\mathrm{PbSnTe}: \mathrm{In}$ определяются состояниями, локализованными на поверхности. Очевидно, что динамика изменения тока исток/сток определяется изменением концентрации свободных носителей заряда, участвующих в экранировке внешнего электрического поля затвора наряду с носителями заряда, локализованными на ловушках на поверхности. В рамках таких упрощенных представлений сильное нарастание тока на рис. 4 при быстром росте положительного затворного напряжения в интервале $t=0-50 \mathrm{c}$ связано с тем, что в этом интервале поле затвора экранируется преимущественно свободными электронами, а сравнительно „медленные“ поверхностные локализованные состояния не успевают захватывать электроны. После прекращения роста $U_{\text {gate }}$ в течение $\sim 450$ с происходит перераспределение экранирующих поле электронов в пользу локализованных на ловушках, так что примерно при $t \approx 500$ с ток уменьшается более чем на 2 порядка. Разная динамика фототока для разных полярностей $U_{\text {gate }}$ на этом же рисунке, очевидно, связана с разной заселенностью ловушек, влияющих на величину фототока. Особенности, наблюдаемые после начала уменьшения $\left|U_{\text {gate }}\right|$ (вблизи $t \approx 900 \mathrm{c})$ находят качественное объяснение, как следствие определяющего влияния на проводимость канала МДП-транзистора после „выключения“ затворного напряжения заряда, локализованного на „медленных“ состояниях на поверхности (BOX charge) [9]. Подчеркнем, что в эксперименте наблюдалось множество эффектов разного типа, включая не затронутые в рамках данной статьи особенности осцилляционного типа и температурные зависимости ЭП. В частности, температурные зависимости ЭП дают основания предполагать, что существенный вклад в наблюдаемые особенности могут давать сегнетоэлектрические свойства PbSnTe: In, а именно доменная структура пленок при гелиевых температурах. Очевидно, что точное количественное описание всего комплекса наблюдаемых особенностей вряд ли возможно. Вместе с тем можно считать доказанным чрезвычайно сильное влияние поверхности на интегральную проводимость изолирующих пленок PbSnTe:In.

\section{4. Заключение}

В пленках PbSnTe:In, выращенных МЛЭ, в эффекте поля впервые наблюдалось гигантское (до $10^{5}$ раз) изменение проводимости в режиме токов, ограниченных пространственным зарядом ТОПЗ со сложной динамикой, определяемой локализованными поверхностными 
состояниями со сложным спектром. Обнаружено сильное влияние состояния поверхности на характеристики исследованной МДП-структуры, позволяющее считать, что значительное число релаксационных особенностей в полуизолирующих тонких пленках $\mathrm{PbSnTe}: \mathrm{In}$ определяется поверхностными состояниями.

\section{Финансирование работы}

Работа выполнена при частичной поддержке РФФИ № 17-02-0575а и РНФ 17-12-01047.

\section{Конфликт интересов}

Авторы заявляют, что у них нет конфликта интересов.

\section{Список литературы}

[1] R. Zhong, X. He, J.A. Schneeloch, C. Zhang, T. Liu, I. Pletikosić, T. Yilmaz, B. Sinkovic, Q. Li, W. Ku, T. Valla, J.M. Tranquada, G. Gu. Phys. Rev. B, 91, 195321 (2015).

[2] V.V. Volobuev, P.S. Mandal, M. Galicka, O. Caha, J. SánchezBarriga, D. Di Sante, A. Varykhalov, A. Khiar, S. Picozzi, G. Bauer, P. Kacman, R. Buczko, O. Rader, G. Springholz. Adv. Mater., 29 (3), 1604185 (2017).

[3] A.E. Klimov, V.N. Sherstyakova, V.N. Shumsky. Ferroelectrics, 378, 101 (2009).

[4] Б.А. Волков, Л.И. Рябова, Д.Р. Хохлов. УФН, 172 (8), 875 (2002).

[5] A.M. Shuvaev, V. Dziom, N.N. Mikhailov, Z.D. Kvon, Y. Shao, D.N. Basov, A. Pimenov. Phys. Rev. B, 96, 155434 (2017).

[6] O.E. Tereshchenko, S.I. Chikichev, A.S. Terekhov, J. Vac. Sci. Technol. A, 17, 2655 (1999).

[7] R. Fu, J. Pattison. Opt. Engin., 51 (10), 104003 (2012).

[8] А.Н. Акимов, А.Э. Климов, А.М. Самойлов, В.Н. Шумский, В.С. Эпов. Конденсированные среды и межфазные границы, 15 (4), 375 (2013).

[9] H.J. Hovel. Sol. St. Electron., 47 (8), 1311 (2003).

Редактор Г.А. Оганесян

\section{Surface conductivity dynamics in PbSnTe: In films in the vicinity of a band inversion}

\author{
A.E. Klimovi,2, A.N. Akimov' , I.O. Akhundov', \\ V.A. Golyashov ${ }^{1,3}$, D.V. Gorshkov ${ }^{1}$, D.V. Ishchenko ${ }^{1}$, \\ G.Yu. Sidorov ${ }^{1}$, S.P. Suprun ${ }^{1}$, A.S. Tarasov ${ }^{1}$, \\ V.S. Epov ${ }^{1}$, O.E. Tereshchenko ${ }^{1,3}$
}

${ }^{1}$ Rzhanov Institute of Semiconductor Physics, Siberian Branch of Russian Academy of Sciences, 630090 Novosibirsk, Russia

${ }^{2}$ Novosibirsk State Technical University, 630073 Novosibirsk, Russia

${ }^{3}$ Novosibirsk State University, 630090 Novosibirsk, Russia

\begin{abstract}
At helium temperatures, the features of transient processes in the field effect without and under illumination in $\mathrm{PbSnTe}$ : In films with current variation up to 105 times are considered. These features qualitatively corresponding to model in which there is a large concentration of traps with different parameters on or near the surface of PbSnTe:In. The role of the surface is confirmed by a strong change in the experimental characteristics after chemical removal of oxides from the PbSnTe:In surface and its passivation with an $\mathrm{Al}_{2} \mathrm{O}_{3}$ layer.
\end{abstract}

